\title{
Chromosome aberrations in astronauts
}

\author{
K. George ${ }^{1}$, M. Durante ${ }^{2}$ and F. A. Cucinotta ${ }^{3}$ \\ ${ }^{1}$ Wyle laboratories, 1290 Hercules Drive, Houston, TX 77058, USA \\ ${ }^{2}$ University Federico II, Department of Physics, Napoli 80126, Italy \\ ${ }^{3}$ NASA Johnson Space Center, SK, 2101 NASA Parkway, Houston, TX 77058, USA
}

\begin{abstract}
A review of currently available data on in vivo induced chromosome damage in the blood lymphocytes of astronauts proves that, after protracted exposure of a few months or more to space radiation, cytogenetic biodosimetry analyses of blood collected within a week or two of return from space provides a reliable estimate of equivalent radiation dose and risk. Recent studies indicate that biodosimetry estimates from single spaceflights lie within the range expected from physical dosimetry and biophysical models, but very large uncertainties are associated with single individual measurements and the total sample population remains low. Retrospective doses may be more difficult to estimate because of the fairly rapid time-dependent loss of "stable" aberrations in blood lymphocytes. Also, biodosimetry estimates from individuals who participate in multiple missions, or very long (interplanetary) missions, may be complicated by an adaptive response to space radiation and/or changes in lymphocyte survival and repopulation. A discussion of published data is presented and specific issues related to space radiation biodosimetry protocols are discussed.
\end{abstract}

\section{Introduction}

Human spaceflight missions have increased in duration over the years. From brief forays lasting up to a few days, missions have extended to months on board the International Space Station (ISS), and future Mars exploration missions could extend as long as three years. This increased time in space has raised concerns about the potential health effects of radiation exposures incurred during missions. Since cancer is the main health concern related to space radiation exposure, limits are based on the lifetime excess cancer risk (Cucinotta and Durante 2006) and are currently restricted to an excess lifetime cancer mortality risk of no more than 3\% (NCRP 2000).

The space radiation environment is a complex mixture of different types of radiation and is very different from the terrestrial radiation environment. The primary sources of radiation in outer space are galactic cosmic rays (GCR), protons and electrons trapped in the Earth's magnetic field, and infrequent solar particle events (SPE). In addition, secondary radiation, such as neutrons and light charged particles, is created by interactions of high-charge (Z) and high-energy (E) nuclei (HZE particles) with spacecraft shielding material and with the human body itself (NCRP 2000). Although HZE particles contribute only a few percent to the particle flux in space, the significantly higher ionizing energy and greater penetration power of this type of radiation could contribute significantly to biological effects.

Health risks to crew members are currently assessed from physical measurements of radiation dose measured using passive thermoluminescent dosimeters (TLDs) and plastic nuclear track detectors (PNTDs). These measurements provide accumulated absorbed skin doses that are combined with computerized anatomical models and radiation transport codes that estimate the effects of shielding. The data are used to calculate radiation doses to specific organs and to assess the corresponding health risk. However, in this process substantial limitations arise that lead to significant uncertainty in estimating 
the biologically important dose (Cucinotta and Durante 2006). Quality factors for space radiation risk coefficients (mostly derived from the A-bomb survivors database), and the dose and dose-rate effectiveness factor, are all quantities affected by large uncertainty. In addition, the location of crew dosimeters can not always be assured, individual radiosensitivity is totally neglected in the risk models, and synergism between microgravity and radiation is still poorly understood. Recently, biodosimetry programs have been introduced that derive equivalent dose or risk from direct measurements of cytogenetic damage in the peripheral blood lymphocytes of crew members (George et al 2001a). These data have the added advantage of taking into account individual radio-sensitivity in addition to the influence of microgravity effects and other stress conditions, and can be very useful to benchmark the current models that are used to estimate risk from physical dosimetry measurements (NCRP 2002).

Cytogenetic biodosimetry methods have been used extensively for assessing radiation exposures, and remain the most sensitive in vivo indicator of dose available to date (IAEA 2001). Dicentric chromosomes have been used for many years to evaluate accidental radiation exposures because they can be easily identified in Giemsa-stained chromosome preparations and pre-exposure background levels are very low. However, the astronaut population is unique in that they consent to considerably higher occupational radiation exposures than terrestrial radiation workers and, unlike the situation with accident victims, pre-exposure levels of chromosome damage can be accurately assessed in astronauts, allowing greater sensitivity in biological dose estimations.

\section{Measured Yields of Exchanges in the Peripheral Blood Lymphocytes of Astronauts}

The first in vivo studies of chromosome damage in the peripheral blood lymphocytes of crew members were conducted in the 1960s during the Gemini program. Bender et al. (1967 and 1968) used solid Giemsa staining techniques to assess dicentrics, rings, fragments, and chromatid aberrations in blood samples obtained from astronauts who participated in the Gemini-III and Gemini-XI missions, which lasted about 5 hours and 3 days, respectively. No significant increase in damage was detected when samples collected before and after flight were compared. More recently, these techniques were tested on crew members participating in longer-duration flights on board the Mir space station (Testard et al. 1996, Obe et al. 1997, Federenko et al. 2001). Testard et al. (1996) studied seven crew members, two of whom participated in flights lasting three weeks or less and had no significant increase in chromosome damage post flight, and five of whom participated in a six-month mission and had increased chromosome damage post flight. Biological dose estimates for the longer-duration flights were calculated using established in vitro calibration curves for $\mathrm{X}$-rays and varied among individuals, with values ranging from 130 mGy to 455 mGy. Obe et al. (1997) studied crew members who participated in space flights lasting from 120 to 198 days, and they reported significant increases in chromosome damage post flight for six of the seven individuals studied. Pooled data for the six individuals who had increased chromosome damage showed a post flight dicentric yield that was roughly 3.5 times that of preflight values. Individual biological doses were estimated using published in vitro X-ray-induced dose-response curves, and values ranged from $50 \mathrm{mGy}$ to $290 \mathrm{mGy}$ depending on the individual. Limited physical dose measurements were reported for the two studies described above, but data indicated that the frequencies of aberrations roughly correlated with absorbed radiation dose. A study by Federenko et al. (2001) of 22 individuals who participated in space missions lasting an average of 4 to 6 months also reported that the mean yields of dicentrics were about 3fold greater after flight than before flight. However, large inter-individual variations were reported for this group, with some individuals having no appreciable increase in dicentric yield post flight. The biological dose estimates for the individual crew members with increased dicentric yields varied from 20 to $280 \mathrm{mGy}$. No comparisons with physical dose estimates were given for this study. 
Evidence from these cytogenetic studies strongly indicates that the radiation dose accumulated during a long-duration flight can induce a measurable increase in the yield of chromosome damage, whereas the effects of doses encountered during shorter missions of a few weeks or less are below the threshold for detection using this technique. However, the type of chromosome aberration evaluated is an important factor when considering these data. All the studies described above focus on the detection of dicentric chromosomes, and it has been well documented that radiation-induced dicentrics in blood lymphocytes undergo time-dependent decay. Dicentrics have been estimated to have an average half-life of about three years in human peripheral blood lymphocytes, but recent studies indicate large variability in decay rate among individuals (Durante et al. 2005). It would follow that the use of dicentric aberrations for biodosimetry analysis after missions lasting several months or more may not be reliable due to the loss of aberrant cells during the mission. In addition, any significant individual variability in the rate of loss of cells with dicentrics would indicate that yields could not be accurately corrected for elimination of this type of exchange from the blood.

The introduction of the fluorescence in situ hybridization (FISH) chromosome painting technique radically improved the ease and accuracy of assessing yields of monocentric aberrations such as translocations (e.g. Lucas 1997). These types of socalled "stable" chromosome aberrations have a higher probability of survival after cell division than unstable aberrations, and are therefore commonly believed to persist in peripheral blood cells for many years. This indicates that analysis of translocations would be a more appropriate tool than dicentric analysis for assessing the effect of chronic radiation exposures such as those encountered during long-duration missions, as well as for assessing retrospective radiation doses. Background levels of translocations are higher than those of dicentrics; they are known to increase with age and show considerable variation between individuals (e.g. Tucker 2001). Although this can be a challenge for situations where pre-exposure levels of translocations are not available, such as after accidental radiation exposure, it is not a problem for space flight biodosimetry because samples can be obtained before flight.

George et al. (2001a) used chromosome painting to assess the yield of chromosome exchanges in the peripheral lymphocytes of six crew members before and after they participated in space missions lasting three months or more and in two crew members who participated in a 10-day shuttle mission. Post flight increases in chromosome damage were detected in all of the crew members on the longer-duration missions, whereas no significant change was detected in crew members on the shorter shuttle mission. Doses were determined using preflight in vitro dose-response curves for gamma rays that were generated using the individual crew members' blood lymphocytes. Preflight levels of translocations were shown to vary considerably between individuals. Doses estimated from total chromosome damage ranged from 31 to $166 \mathrm{mGy}$, depending on the individual, and these values roughly correlated with the physical measurements of dose. Average relative biological effectiveness (RBE) was estimated by comparing biological dose estimates with measured physical doses. Average RBE values were in excellent agreement with the average quality factor of 2.5 that was calculated from physical readings corrected for the HZE component of the exposure and adjusted to representative doses at organ locations. These data confirmed the results of an earlier study by Yang et al. (1997) in which FISH chromosome painting was used to assess aberrations in three crew members who participated in a space mission lasting 115 days.

\section{Biologically Based Risk Estimates}

A direct comparison of risk estimates based on physical dose measurements and application of models (NCRP 2000) with estimates based on biological measurements is possible because chromosome aberrations in peripheral blood lymphocytes have been validated as biomarkers of cancer risk. Risk projections for long-term missions in low Earth orbit (LEO) can be obtained from biodosimetry data using the quantitative 
approach derived from the European Study Group in Cytogenetic Biomarkers and Health (ESCH) database, and the proportion of risk attributable to space radiation was found to be around 23-24\% (Bonassi et al. 2005). The post flight relative increase in aberration frequency can then be used directly to estimate the cancer risk, and this biologically based estimate can be compared with conventional risk estimates based on physical dosimetry and models (NCRP 2000). Through analysis of the cytogenetic data described above, it has been determined that the average excess relative risk (ERR) of cancer induction after long-term missions in LEO is 0.09, with large inter-individual variations (ranging from -0.08 to 0.42 ) (Durante et al. 2001, Durante 2005). Individual risk estimates in this same cohort using conventional assessment leads to an average ERR of 0.05, with a range from 0.02 to 0.12 . Despite the large spread in the "biological" values, it is remarkable that the two estimates lie in the same range. In comparison with calculations of equivalent doses in the NASA study (George et al. 2001a), these results suggest that current average risk estimates for astronauts in LEO are accurate to within a factor of 3 or less (Durante 2005). ERR estimates derived from chromosome aberrations present a wider scatter than those calculated from doses. Certainly, statistical error involving aberration measurements accounts for most of the widened distribution, but inter-individual variability in risk may also contribute to this variance.

\section{Cytogenetic Signatures of Space Radiation Exposure}

In addition to detecting simple reciprocal-type chromosome exchanges, chromosome painting can be used to detect complex-type exchanges, defined as exchanges involving three or more breaks in two or more chromosomes. Many studies have demonstrated that after low-linear energy transfer (LET) radiation such as gamma rays and X-rays at exposures below $2 \mathrm{~Gy}$, no significant increase in complex exchanges is detected, whereas low doses of high-LET radiation, such as the HZE ions in space, are capable of producing extremely complex patterns of chromosome damage (George et al. 2003, Durante et al. 2002). This evidence suggests that complex-type damage can be induced by a single particle's traversal of a cell and indicates that this type of damage can be a biomarker of exposure to low doses of high-LET radiation (Anderson et al. 2002). It follows, then, that any increase in complex-type chromosome damage detected in blood lymphocytes of crew members after space flight would be due entirely to the high-LET component of the exposure.

However, one confounding factor in the assessment of high-LET radiation-induced chromosome damage is the influence of delays in progression of the cell cycle from G2 to metaphase. Recent ground-based studies have indicated that high-LET-induced chromosome damage undergoes a complicated pattern of mitotic delay (George et al. 2001b), and it is assumed that this is due to the less homogenous nature of this type of exposure compared with X-ray or gamma-ray irradiation. When chromosomes in the G2 phase of the cell cycle are used for the analysis of high-LET-induced cytogenetic damage, much higher yields of damage are obtained than with standard metaphase analysis. This is due to extensive G2-metaphase cell cycle delays. It is therefore possible that post flight expression of complex chromosome damage in crew members' lymphocytes could be influenced by a complicated pattern of mitotic delay after exposure to the mixed radiation field encountered in space, and consequently it could be underestimated in metaphase samples collected at one time point. To ensure that chromosome damage is not underestimated in astronauts' blood lymphocytes, it may be more appropriate to use the chemically induced premature chromosome condensation (PCC) technique, in which chromosomes in the G2 phase as well as metaphase are assessed.

Cytogenetic analysis using FISH painting of chromosomes in lymphocytes from ten astronauts after long-term space missions of three months or more revealed that the frequency of complex damage was very low and in most cases only one or two complex exchanges were detected in several thousand PCC samples analyzed for each individual (George et al. 2003). A low background level of complex damage was also detected in preflight samples, hampering statistical significance. Similar results were obtained in a 
cohort of Russian cosmonauts (Durante et al. 2004). In another study, Horstmann et al. (2005) used a multiplex FISH technique (MFISH) to assess all chromosomes simultaneously in each cell and detected no complex-type exchanges in a total of 3590 lymphocyte cells pooled from astronauts involved in short- and long-duration space missions. However, this study was conducted using standard metaphase analysis and therefore does not discount the possibility that complex damage was not expressed due to extensive cell-cycle delay. In addition, both Testard et al. (1996) and Obe et al. (1997) reported a very low frequency of highly damaged metaphase cells after long-duration missions. However, because analysis was conducted using solid staining techniques, these studies were limited to so-called "rogue" cells containing multiple dicentric and acentric fragments, and again cells were analyzed using standard metaphase techniques. The nature of the "rogue" cells is still unclear, but they are generally considered to be unrelated to radiation exposure (Mustonen et al. 1998).

Intrachromosomal exchanges (inversions) have also been suggested as a biomarker of exposure to densely ionizing radiation (Hande et al. 2003). Only one group so far (Horstmann et al. 2005) has attempted to assess the frequency of intrachromosomal exchanges in blood lymphocytes after space flight. For this study, a high-resolution multicolor fluorescence banding (mBAND) probe for chromosome 5 was used, and no inversions were detected in 2800 cells pooled from 11 astronauts involved in short- and long-duration missions.

\section{Persistence of Space Radiation-Induced Cytogenetic Damage}

It has been commonly believed that chromosome translocations would persist in the blood lymphocytes for many years. However, as more data become available, evidence suggests that this may not always be the case (reviewed in Tucker 2001, IAEA 2001, Durante 2005). Some limited data are available on the persistence of chromosome aberrations in astronaut peripheral blood lymphocytes. Durante et al. (2003) clearly demonstrated that dicentric yields in astronauts decay with time after flight. The authors could not measure the complete time dependence of translocations using FISH painting, but their results show a lack of correlation between time in space and translocation yields (figure 1A.

No significant correlation was observed between yields of dicentrics or translocations and duration of flight or absorbed dose. The yield of aberrations declined rapidly during the time interval between flights, and after multiple flights translocation yields were similar to background levels before the first mission (figure 1B).

George et al. (2005) analyzed blood samples collected at various times, beginning directly after return from space to more than 5 years after flight. For five of the six astronauts studied, the analysis of individual time courses for translocations revealed a temporal decline of yields, with half-lives ranging from 10 to 58 months. Since the level of stable aberrations depends on the interplay between natural loss of circulating $\mathrm{T}$ lymphocytes and replenishment from the stem or progenitor cells, the differences in the rates of decay could be explained by inter-individual variation in lymphocyte turnover. The results indicate possible complications with the use of stable aberrations for retrospective dose reconstruction or risk estimates from biological data. It should be stressed that space is a unique environment and these results may not be applicable to terrestrial radiation exposures. It is possible that confounding factors in the space environment, such as stress and microgravity, could induce cell instability. It has been shown that space flights induce drastic changes in the immune system, and the ability to respond to challenges such as infection is reduced in the space environment.

The complex time dependence of the yield of chromosome aberrations is apparently a severe limitation on the use of cytogenetic endpoints to estimate equivalent dose or risk. The result of this biological test will depend strongly on the time after sampling. However, it can be argued that the "time factor" does not necessarily reflect a technical limitation of the cytogenetic biomarker, but rather provides an individual risk assessment rather than the average risk estimate based solely on absorbed dose (Durante et al. 2005). 
As in the case of individuals who quit smoking at a certain age after years of chronic exposure to carcinogens in cigarette smoke, relative risk from radiation decreases with time after exposure (as shown in the recent analysis of A-bomb survivors, see Preston et al. 2003), and the decrease is likely to show a large inter-individual variability, caused by physiological, environmental, and genetic factors. Since these factors are not taken into account in biophysical models of radiation action, a reliable picture of the relative risk for an individual at a given time after exposure may be derived only by biomarkers of risk.

\section{Methodology Considerations and Suggestions for Standardized Protocols}

After all available data are considered, it appears that cytogenetic biodosimetry can be used reliably to reconstruct radiation dose after protracted exposure to space radiation, and these data can potentially be used to assess individual health risk. However, it is becoming clear that compared with biodosimetry of terrestrial radiation exposure, biodosimetry of space radiation exposure has some unique challenges, which must be addressed before a standard procedure can be established. It is apparent that several major areas should be considered, as addressed below.

\subsection{Collection of Chromosome Samples}

Lymphocytes must be examined in blood samples that were collected before flight to establish accurate preflight yields of damage. This not only provides an individual baseline for translocations, which are highly variable between individuals, but also increases the dose sensitivity of the assay. Considering that chromosome damage may undergo a fairly rapid time-dependent decay, post flight samples must be collected within a week or two after the mission. Some studies have used samples collected the day after return from space. However, it has been noted that the response of lymphocytes to phytohemagglutinin (PHA), a chemical that is used to induce lymphocytes to undergo cell division, is severely suppressed on the day of return from space. The mitotic index was drastically lower in samples collected from three crew members on the day of return from a 3-month space mission than it was in preflight samples collected from the same individuals (Yang et al. 1997). The number of cycling cells was much lower, and progression through the cell cycle was much slower. This phenomenon, which may be related to changes in the immune response and/or other stress and microgravity effects, seems to last only a few days. The growth kinetics of PHA-stimulated lymphocytes collected 9 days after flight were almost the same as those in preflight samples. Given the challenges of collecting chromosome samples within a few days of return from flight, a later sampling time (7-14 days after flight) is preferable.

Samples that have been subjected to chemically induced PCC contain G2-phase as well as metaphase cells. Analysis of such samples is preferable to standard metaphase analysis. PCC analysis will ensure that any cells undergoing extensive G2-M cell-cycle delay will still be included in the analysis. As mentioned above, this method has been shown to help avoid population bias after high-LET radiation in vitro and may ensure correct yields of complex damage induced in vivo, although this has yet to be proven. In addition, PCC samples generally have a higher index of analyzable cells, so that using them makes it easier to obtain sufficient cells from samples that are consistently difficult to culture.

\subsection{Assessment of Chromosome Damage}

Since it has been well documented that radiation-induced dicentrics undergo fairly fast time-dependent decay and will certainly be lost from the blood during a longer spaceflight mission, stable monocentric aberrations will provide more accurate assessments of dose. A combination of three FISH painting probes for the larger chromosome pairs that comprise about $20 \%$ of the genome is recommended for analysis. This technique allows damage to be scored quickly, and if each chromosome is painted with a different color, the detection of complex damage will be maximized while 
exchanges within the painted chromosomes can also be detected. Data for analysis of individual combinations of chromosomes can be extrapolated to whole-genome equivalent values using the appropriate calculations (for example, see George et al. 2001a). Use of MFISH techniques is not recommended for routine biodosimetry analysis because analysis of individual cells takes a considerable amount of time.

The analysis of complex-type exchanges could provide valuable information about the high-LET component of radiation exposure, as any significant increase will likely be due to heavy ions. However, it appears this type of exchange is quite rare and it is unlikely that valuable data can be obtained using the average number of cells analyzed for these tests.

One other important consideration when scoring chromosome damage for dose estimations is that clonal exchanges must be identified and excluded. Several recent studies have indicated that some individuals' blood lymphocytes possess cells with balanced chromosome abnormalities that have clonally expanded in vivo, resulting in a population of cells with identical rearrangements. Including these clonal exchanges can result in an overestimation of radiation-induced damage and therefore influence the accuracy of dose calculations. Clonal exchanges may be more prevalent than previously thought. In fact, in one study clonal aberrations were identified in three of twelve astronauts (George et al 2004). These clonal cells were present in samples collected both before and after space flight, and yields were higher than those previously reported for healthy individuals in this age range (40-52 years of age). The individuals with clonal aberrations were followed over several months, and the yields of all clones decreased during this period. It is possible that the inclusion of clonal exchanges could explain some of the inter-individual biological differences in dose estimates for astronauts. Since clonal aberrations may be associated with increased risk of tumorigenesis, it is important for risk assessment as well as biodosimetry to accurately identify cells containing clonal rearrangements.

\subsection{Dose Limitations}

The radiation dose incurred during shorter space flight missions does not produce effects detectable by currently available cytogenetic biodosimetry techniques. The lowest dose that can be assessed seems to be around $10 \mathrm{cGy}$. This generally restricts the use of biodosimetry to missions lasting at least 3 months. Using the FISH technique, several thousand cells should be analyzed in both pre- and post flight samples to obtain statistically significant data, although exact numbers can vary depending on the individual's background frequencies of damage.

\subsection{Individual Sensitivity}

Recent data indicate that in vitro dose response for chromosome damage may vary for some individuals (figure 2). If this is the case, then one standard calibration curve can not be used for all individuals and it is necessary to generate a preflight in vitro calibration curve for blood lymphocytes from each crew member before their participation in the mission. This will indicate if the particular individual has a greater predisposition for sensitivity to radiation and should be monitored more closely. In addition, if the same combination of chromosomes is assessed before and after flight and individual dose-response curves are used, then the results can be compared directly without the need for subtracting background yield of chromosome damage. This results in substantially decreased error in dose estimations.

Recent estimates have put the overall percentage of individuals heterozygous for the ataxia telangiectasia (A-T) gene at around 3\% of the human population. These individuals have defective DNA repair capabilities that make them more sensitive to radiation, and they are clearly at greater risk of health effects due to radiation exposure. If these percentages are correct, it is very likely that the astronaut population includes some A-T heterozygous individuals. Clearly the issue of individual sensitivity to space radiation is very important and data are limited. More study is required in this area. 


\subsection{Dose Estimations}

As mentioned above, if the same combination of chromosomes is assessed for each sample, then post flight increases can be obtained by subtracting preflight background yields from the post flight yield, and biologically determined dose can be calculated directly using the curve fit for the preflight calibration curve. At least two doses below 10 cGy should be used for the in vitro calibration to increase the accuracy of the curve-fitting for doses within the range encountered during space flight. At least five different doses are required for the calibration curve, and using the probes that comprise $20 \%$ of the whole genome, several thousand cells must be analyzed to generate sufficient data for curve-fitting.

\subsection{Influence of Multiple Flights}

The recent study of crew members after multiple flights (Durante et al. 2003) indicates that individuals who have been involved in several missions may have an adaptive response to space radiation exposure. Given that these individuals may have less of an increase in chromosome damage than would be expected after multiple flights, it may not be possible to accurately assess their dose using these methods. It is important, therefore, to research an individual's previous radiation exposure from earlier space flights and possibly medical procedures, and to take this into consideration when evaluating biodosimetry data. This study of adaptive response is very interesting and it is important that the phenomenon be investigated further.

\section{References}

Anderson, R.M., Stevens, D.L., Goodhead, D.T. M-FISH analysis shows that complex chromosome aberrations induced by alpha -particle tracks are cumulative products of localized rearrangements. Proc. Natl. Acad. Sci. USA 99, 12167-12172, 2002.

Bender, M.A., Gooch, P.C., Kondo, S. The Gemini-3 S-4 spaceflight-radiation interaction experiment. Radiat. Res. 31, 91-111, 1967.

Bender, M.A, Gooch, P.C., Kondo, S. The Gemini XI S-4 spaceflight-radiation interaction experiment: the human blood experiment. Radiat. Res. 34, 228-238, 1968.

Bonassi, S., Ugolini, D., Kirsh-Volders, M., Stromberg, U., Vermeulen, R., Tucker, J.D. Human population studies with cytogenetic biomarkers: review of the literature and future prospectives. Environ. Mol. Mutagen 45, 258-270, 2005.

Cucinotta, F.A., Durante, M. Cancer risk from exposure to galactic cosmic rays: implications for space exploration by human beings. Lancet Oncol. 7, 431-435, 2006.

Durante, M. Biomarkers of space radiation risk. Radiat. Res. 164, 467-473, 2005.

Durante, M., Bonassi, S., George, K., Cucinotta, F.A. Risk estimation based on chromosomal aberrations induced by radiation. Radiat. Res. 156, 662-667, 2001.

Durante, M., George, K., Wu, H., Cucinotta, F.A. Karyotypes of human lymphocytes exposed to high-energy iron ions. Radiat. Res. 158, 581-590, 2002.

Durante, M., Snigiryova, G., Akaeva, E., Bogomazova, S., Druzhinin, S., Federenko, B., Greco, O., Novitskaya, N., Rubanovich, A., Shevchenko, V., von Recklinghausen, U., 
Obe, G. Chromosome aberration dosimetry in cosmonauts after single or multiple flights. Cytogenet. Genome Res. 103, 40-46, 2003.

Durante, M., Ando, K., Furusawa, Y., Obe, G., George, K., Cucinotta, F.A. Complex chromosomal rearrangements induced in vivo by heavy ions. Cytogenet. Genome Res. 104, 240-244, 2004.

Federenko, B., Druzhinin, S., Yudaeva, L., Petrov, V., Akatov, Y., Snigiryova, G., Novitskaya, N., Shevchenko, V., Rubanovich, A. Cytogenetic studies of blood lymphocytes from cosmonauts after long-term space flights on Mir station. Adv. Space Res. 27, 355-359, 2003.

George, K., Durante, M., Wu, H., Willingham, V., Badhwar, G., Cucinotta, F.A. Chromosome aberrations in astronauts' blood lymphocytes after space flight. Radiat. Res. 156, 731-738, 2001a.

George, K., Wu, H., Willingham, V., Furusawa, Y., Kawata, T., Cucinotta, F.A. Highand low-LET induced chromsome damage in human lymphocytes: a time course of aberrations in metaphase and interphase. Int. J. Radiat. Biol. 77, 175-183, 2001 b.

George, K., Wu, H., Willingham, V., Cucinotta, F.A. The effect of space radiation on the induction of chromosome damage. Physica. Medica, 17, 224-228, 2001c.

George, K., Durante, M., Willingham, V., Wu, H., Yang, T.C., Cucinotta, F.A. Biological effectiveness of accelerated particles for the induction of chomosome damage measured in metaphase and interphase human lymphoyctes. Radiat. Res. 160, 425-435, $2003 a$.

George, K., Durante, M., Wu, H., Willingham, V., Cucinotta, F.A. In vivo and in vitro measurements of complex-type chromosomal exchanges induced by heavy ions. Adv. Space Res. 31, 1525-1535, 2003b.

George, K., Durante, M., Willingham, V., Cucinotta, F.A. Chromosome aberrations of clonal origin are present in astronauts' blood lymphocytes. Cytogenet. Genome Res. 104, 245-251, 2004.

George, K., Willingham, V., Cucinotta, F.A. Stability of chromosome aberrations in the blood lymphocytes of astronauts after space flight by FISH chromosome painting. Radiat. Res. 164, 474-480, 2005.

Hortsmann, M., Durante, M., Johannes, C., Pieper, R., Obe, G. Space radiation does not induce a significant increase of intrachromosomal exchanges in astronauts' lymphocytes. Radiation Environ. Biophysics 44, 219-224, 2005.

IAEA Cytogenetic Analysis for radiation dose assessment. Technical Report No. 405. International Atomic Energy Agency, Vienna, 2001.

Lucas, J.N. Dose reconstruction for individuals exposed to ionizing radiation using chromosome painting. Radiat. Res. 148, 33-38, 1997.

Mustonen, R., Lindholm, C., Tawn, E.J., Sabatier, L., Salomaa, S. The incidence of cytogenetically abnormal rogue cells in peripheral blood. Int. J. Radiat. Biol. 74, 781-785, 1998.

NCRP Radiation Protection Guidance for Activities in Low-Earth Orbit. NCRP Report No. 132, Bethesda, Maryland, 2000. 
NCRP Operational Radiation Safety Program for Astronauts in Low-Earth Orbit: a Basic Framework. NCRP Report No. 142, Bethesda, Maryland, 2002.

Obe, G., Johannes, I., Johannes, C., Hallman, K., Reitz, G., Facius, R. Chromosomal aberrations in blood lymphocytes of astronauts after long-term space flights. Int. J. Radiat. Biol. 72, 727-734, 1997.

Preston, D.L., Pierce, D.A., Shimizu, Y., Ron, E., Mabuchi, K. Dose response and temporal patterns of radiation-associated solid cancer risks. Health Phys. 85, 43-46, 2003.

Testard, L., Ricoul, M., Hoffschir, F., Flury-Herard, A., Dutrillaux, B., Federenko, B., Gerasimenko, V., Sabatier, L. Radiation-induced chromosome damage in astronauts' lymphocytes. Int. J. Radiat. Biol. 70, 403-411, 1996.

Tucker, J.D. FISH cytogenetics and the future of radiation biodosimetry. Radiat. Prot. Dosimetry 97, 55-60, 2001.

Yang, T.C., George, K., Johnson, A.S., Durante, M., Fedorenko, B.S. Biodosimetry results from space flight Mir-18. Radiat. Res. 48, 17-23, 1997. 
Figure Captions

Figure 1. Time course of chromosome aberration in a study on 22 Russian cosmonauts. A. Relationship between translocation frequency (WGE) and total duration of space sojourns for cosmonauts involved in multiple space flights. Each symbol represents a different cosmonaut. B. Kinetics of dicentrics in one cosmonaut involved in 4 spaceflights. The dicentric frequency (per 1000 lymphocytes) is plotted vs. the time from the first blood draw used for the cytogenetic test. Vertical bars indicate the times of launch and landing for the mission, and the number between the bars indicates the flight number for the cosmonaut. The line connecting the datapoints is a guide for the eye. Plots from reference Durante et al. (2003), reproduced with permission of Karger Publishers, Basel.

Figure 2. Dose-response curves for chromosome damage induced after in vitro irradiation with gamma rays. Each symbol represents a different crew member. Bars represent standard error. 


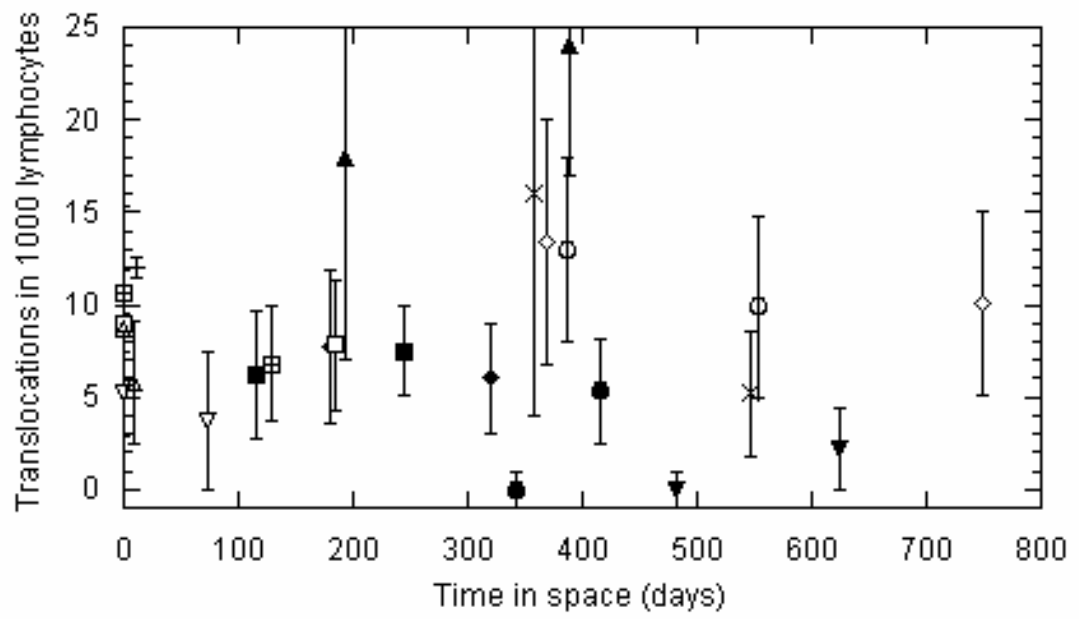



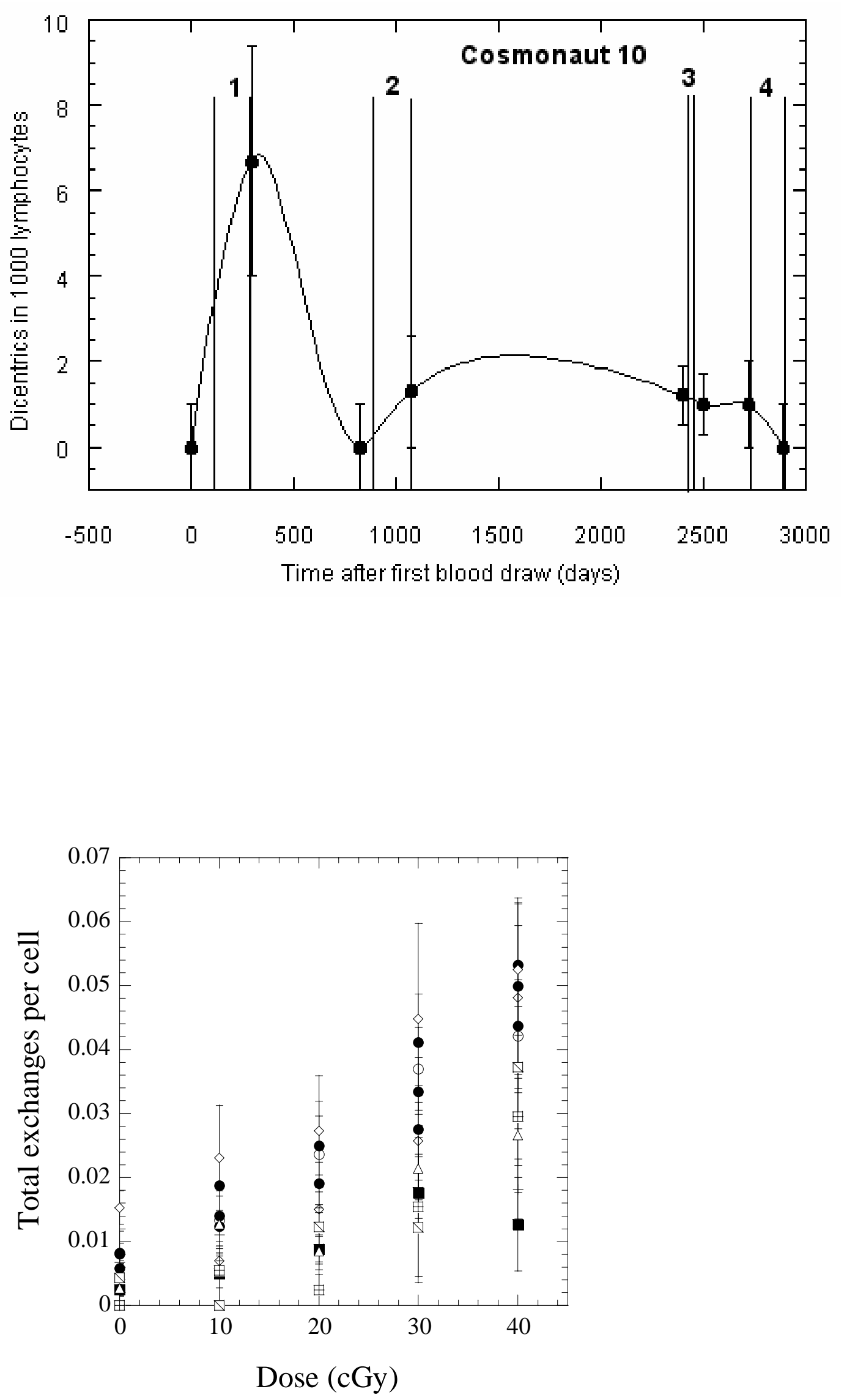